\title{
Note on certain inequalities for Neuman means
}

\author{
Shu-Bo Chen ${ }^{1}$, Zai-Yin He², Yu-Ming Chu ${ }^{1 *}$, Ying-Qing Song ${ }^{1}$ and Xiao-Jing Tao ${ }^{3}$
}

"Correspondence:

chuyuming2005@126.com

'School of Mathematics and Computation Science, Hunan City University, Yiyang, 413000, China Full list of author information is available at the end of the article

\begin{abstract}
In this paper, we give the explicit formulas for the Neuman means $N_{A H}, N_{H A}, N_{A C}$, and $N_{C A}$, and present the best possible upper and lower bounds for these means in terms of the combinations of harmonic mean $H$, arithmetic mean $A$, and contraharmonic mean $C$.
\end{abstract}

MSC: 26E60

Keywords: Schwab-Borchardt mean; Neuman mean; harmonic mean; arithmetic mean; contraharmonic mean

\section{Introduction}

Let $a, b, c \geq 0$ with $a b+a c+b c \neq 0$. Then the symmetric integral $R_{F}(a, b, c)[1]$ of the first kind is defined as

$$
R_{F}(a, b, c)=\frac{1}{2} \int_{0}^{\infty}[(t+a)(t+b)(t+c)]^{-1 / 2} d t .
$$

The degenerate case of $R_{F}$, denoted by $R_{C}$, plays an important role in the theory of special functions $[1,2]$, which is given by

$$
R_{C}(a, b)=R_{F}(a, b, b)
$$

For $a, b>0$ with $a \neq b$, the Schwab-Borchardt mean $\operatorname{SB}(a, b)$ [3-5] of $a$ and $b$ is given by

$$
\operatorname{SB}(a, b)= \begin{cases}\frac{\sqrt{b^{2}-a^{2}}}{\cos ^{-1}(a / b)}, & a<b \\ \frac{\sqrt{a^{2}-b^{2}}}{\cosh ^{-1}(a / b)}, & a>b\end{cases}
$$

where $\cos ^{-1}(x)$ and $\cosh ^{-1}(x)=\log \left(x+\sqrt{x^{2}-1}\right)$ are the inverse cosine and inverse hyperbolic cosine functions, respectively.

Carlson [6] (see also [7, (3.21)]) proved that

$$
\operatorname{SB}(a, b)=\left[R_{C}\left(a^{2}, b^{2}\right)\right]^{-1}
$$

Recently, the Schwab-Borchardt mean has been the subject of intensive research. In particular, many remarkable inequalities for the Schwab-Borchardt mean and its generated means can be found in the literature [3-5, 8-11].

C2014 Chen et al.; licensee Springer. This is an Open Access article distributed under the terms of the Creative Commons Attribution License (http://creativecommons.org/licenses/by/2.0), which permits unrestricted use, distribution, and reproduction in any medium, provided the original work is properly cited. 
Let $a>b>0, v=(a-b) /(a+b) \in(0,1), p \in(0, \infty), q \in(0, \pi / 2), r \in(0, \log (2+\sqrt{3}))$, and $s \in(0, \pi / 3)$ be the parameters such that $1 / \cosh (p)=\cos (q)=1-v^{2}, \cosh (r)=\sec (s)=$ $1+v^{2}, H(a, b)=2 a b /(a+b), G(a, b)=\sqrt{a b}, A(a, b)=(a+b) / 2, Q(a, b)=\sqrt{\left(a^{2}+b^{2}\right) / 2}$, and $C(a, b)=\left(a^{2}+b^{2}\right) /(a+b)$ be, respectively, the harmonic, geometric, arithmetic, quadratic, and contraharmonic means of $a$ and $b, S_{A H}(a, b)=\mathrm{SB}[A(a, b), H(a, b)]$, $S_{H A}(a, b)=\mathrm{SB}[H(a, b), A(a, b)], S_{A C}(a, b)=\mathrm{SB}[A(a, b), C(a, b)], S_{C A}(a, b)=\mathrm{SB}[C(a, b)$, $A(a, b)]$. Then Neuman [10] gave the explicit formulas

$$
\begin{array}{ll}
S_{A H}(a, b)=A(a, b) \frac{\tanh (p)}{p}, & S_{H A}(a, b)=A(a, b) \frac{\sin q}{q}, \\
S_{C A}(a, b)=A(a, b) \frac{\sinh (r)}{r}, & S_{A C}(a, b)=A(a, b) \frac{\tan s}{s} .
\end{array}
$$

Very recently, Neuman [12] found a new mean $N(a, b)$ derived from the SchwabBorchardt mean as follows:

$$
N(a, b)=\frac{1}{2}\left[a+\frac{b^{2}}{\mathrm{SB}(a, b)}\right]
$$

Let $N_{A H}(a, b)=N[A(a, b), H(a, b)], N_{H A}(a, b)=N[H(a, b), A(a, b)], N_{A G}(a, b)=N[A(a, b)$, $G(a, b)], \quad N_{G A}(a, b)=N[G(a, b), A(a, b)], \quad N_{A C}(a, b)=N[A(a, b), C(a, b)], \quad N_{C A}(a, b)=$ $N[C(a, b), A(a, b)], N_{A Q}(a, b)=N[A(a, b), Q(a, b)]$, and $N_{Q A}(a, b)=N[Q(a, b), A(a, b)]$ be the Neuman means. Then Neuman [12] proved that

$$
G(a, b)<N_{A G}(a, b)<N_{G A}(a, b)<A(a, b)<N_{Q A}(a, b)<N_{A Q}(a, b)<Q(a, b)
$$

for all $a, b>0$ with $a \neq b$, and the double inequalities

$$
\begin{aligned}
& \alpha_{1} A(a, b)+\left(1-\alpha_{1}\right) G(a, b)<N_{G A}(a, b)<\beta_{1} A(a, b)+\left(1-\beta_{1}\right) G(a, b), \\
& \alpha_{2} Q(a, b)+\left(1-\alpha_{2}\right) A(a, b)<N_{A Q}(a, b)<\beta_{2} Q(a, b)+\left(1-\beta_{2}\right) A(a, b), \\
& \alpha_{3} A(a, b)+\left(1-\alpha_{3}\right) G(a, b)<N_{A G}(a, b)<\beta_{3} A(a, b)+\left(1-\beta_{3}\right) G(a, b), \\
& \alpha_{4} Q(a, b)+\left(1-\alpha_{4}\right) A(a, b)<N_{Q A}(a, b)<\beta_{4} Q(a, b)+\left(1-\beta_{4}\right) A(a, b)
\end{aligned}
$$

hold for all $a, b>0$ with $a \neq b$ if and only if $\alpha_{1} \leq 2 / 3, \beta_{1} \geq \pi / 4, \alpha_{2} \leq 2 / 3, \beta_{2} \geq$ $(\pi-2) /[4(\sqrt{2}-1)]=0.689 \ldots, \alpha_{3} \leq 1 / 3, \beta_{3} \geq 1 / 2, \alpha_{4} \leq 1 / 3$, and $\beta_{4} \geq[\log (1+\sqrt{2})+$ $\sqrt{2}-2] /[2(\sqrt{2}-1)]=0.356 \ldots$

Zhang et al. [13] presented the best possible parameters $\alpha_{1}, \alpha_{2}, \beta_{1}, \beta_{2} \in[0,1 / 2]$ and $\alpha_{3}, \alpha_{4}, \beta_{3}, \beta_{4} \in[1 / 2,1]$ such that the double inequalities

$$
\begin{aligned}
& G\left(\alpha_{1} a+\left(1-\alpha_{1}\right) b, \alpha_{1} b+\left(1-\alpha_{1}\right) a\right)<N_{A G}(a, b)<G\left(\beta_{1} a+\left(1-\beta_{1}\right) b, \beta_{1} b+\left(1-\beta_{1}\right) a\right), \\
& G\left(\alpha_{2} a+\left(1-\alpha_{2}\right) b, \alpha_{2} b+\left(1-\alpha_{2}\right) a\right)<N_{G A}(a, b)<G\left(\beta_{2} a+\left(1-\beta_{2}\right) b, \beta_{2} b+\left(1-\beta_{2}\right) a\right), \\
& Q\left(\alpha_{3} a+\left(1-\alpha_{3}\right) b, \alpha_{3} b+\left(1-\alpha_{3}\right) a\right)<N_{Q A}(a, b)<Q\left(\beta_{3} a+\left(1-\beta_{3}\right) b, \beta_{3} b+\left(1-\beta_{3}\right) a\right), \\
& Q\left(\alpha_{4} a+\left(1-\alpha_{4}\right) b, \alpha_{4} b+\left(1-\alpha_{4}\right) a\right)<N_{A Q}(a, b)<Q\left(\beta_{4} a+\left(1-\beta_{4}\right) b, \beta_{4} b+\left(1-\beta_{4}\right) a\right)
\end{aligned}
$$

hold for all $a, b>0$ with $a \neq b$. 
In [14], the authors found the greatest values $\alpha_{1}, \alpha_{2}, \alpha_{3}, \alpha_{4}, \alpha_{5}, \alpha_{6}, \alpha_{7}, \alpha_{8}$, and the least values $\beta_{1}, \beta_{2}, \beta_{3}, \beta_{4}, \beta_{5}, \beta_{6}, \beta_{7}, \beta_{8}$ such that the double inequalities

$$
\begin{aligned}
& A^{\alpha_{1}}(a, b) G^{1-\alpha_{1}}(a, b)<N_{G A}(a, b)<A^{\beta_{1}}(a, b) G^{1-\beta_{1}}(a, b) \text {, } \\
& \frac{\alpha_{2}}{G(a, b)}+\frac{1-\alpha_{2}}{A(a, b)}<\frac{1}{N_{G A}(a, b)}<\frac{\beta_{2}}{G(a, b)}+\frac{1-\beta_{2}}{A(a, b)}, \\
& A^{\alpha_{3}}(a, b) G^{1-\alpha_{3}}(a, b)<N_{A G}(a, b)<A^{\beta_{3}}(a, b) G^{1-\beta_{3}}(a, b) \text {, } \\
& \frac{\alpha_{4}}{G(a, b)}+\frac{1-\alpha_{4}}{A(a, b)}<\frac{1}{N_{A G}(a, b)}<\frac{\beta_{4}}{G(a, b)}+\frac{1-\beta_{4}}{A(a, b)}, \\
& Q^{\alpha_{5}}(a, b) A^{1-\alpha_{5}}(a, b)<N_{A Q}(a, b)<Q^{\beta_{5}}(a, b) A^{1-\beta_{5}}(a, b), \\
& \frac{\alpha_{6}}{A(a, b)}+\frac{1-\alpha_{6}}{Q(a, b)}<\frac{1}{N_{A Q}(a, b)}<\frac{\beta_{6}}{A(a, b)}+\frac{1-\beta_{6}}{Q(a, b)}, \\
& Q^{\alpha_{7}}(a, b) A^{1-\alpha_{7}}(a, b)<N_{Q A}(a, b)<Q^{\beta_{7}}(a, b) A^{1-\beta_{7}}(a, b), \\
& \frac{\alpha_{8}}{A(a, b)}+\frac{1-\alpha_{8}}{Q(a, b)}<\frac{1}{N_{Q A}(a, b)}<\frac{\beta_{8}}{A(a, b)}+\frac{1-\beta_{8}}{Q(a, b)}
\end{aligned}
$$

hold for all $a, b>0$ with $a \neq b$.

The main purpose of this paper is to give the explicit formulas for the Neuman means $N_{A H}, N_{H A}, N_{A C}$, and $N_{C A}$, and to present the best possible upper and lower bounds for these means in terms of the combinations of harmonic, arithmetic, and contraharmonic means. Our main results are Theorems 1.1-1.3.

Theorem 1.1 Let $a>b>0, v=(a-b) /(a+b) \in(0,1), p \in(0, \infty), q \in(0, \pi / 2), r \in$ $(0, \log (2+\sqrt{3}))$, and $s \in(0, \pi / 3)$ be the parameters such that $1 / \cosh (p)=\cos (q)=1-v^{2}$, $\cosh (r)=\sec (s)=1+v^{2}$. Then we have

$$
\begin{aligned}
& N_{A H}(a, b)=\frac{1}{2} A(a, b)\left[1+\frac{2 p}{\sinh (2 p)}\right], \\
& N_{H A}(a, b)=\frac{1}{2} A(a, b)\left[\cos (q)+\frac{q}{\sin (q)}\right], \\
& N_{C A}(a, b)=\frac{1}{2} A(a, b)\left[\cosh (r)+\frac{r}{\sinh (r)}\right], \\
& N_{A C}(a, b)=\frac{1}{2} A(a, b)\left[1+\frac{2 s}{\sin (2 s)}\right],
\end{aligned}
$$

and

$$
\begin{aligned}
H(a, b) & <N_{A H}(a, b)<N_{H A}(a, b)<A(a, b) \\
& <N_{C A}(a, b)<N_{A C}(a, b)<C(a, b) .
\end{aligned}
$$

\section{Theorem 1.2 The double inequalities}

$$
\begin{aligned}
& \alpha_{1} A(a, b)+\left(1-\alpha_{1}\right) H(a, b)<N_{A H}(a, b)<\beta_{1} A(a, b)+\left(1-\beta_{1}\right) H(a, b), \\
& \alpha_{2} A(a, b)+\left(1-\alpha_{2}\right) H(a, b)<N_{H A}(a, b)<\beta_{2} A(a, b)+\left(1-\beta_{2}\right) H(a, b),
\end{aligned}
$$




$$
\begin{aligned}
& \alpha_{3} C(a, b)+\left(1-\alpha_{3}\right) A(a, b)<N_{C A}(a, b)<\beta_{3} C(a, b)+\left(1-\beta_{3}\right) A(a, b), \\
& \alpha_{4} C(a, b)+\left(1-\alpha_{4}\right) A(a, b)<N_{A C}(a, b)<\beta_{4} C(a, b)+\left(1-\beta_{4}\right) A(a, b)
\end{aligned}
$$

hold for all $a, b>0$ with $a \neq b$ if and only if $\alpha_{1} \leq 1 / 3, \beta_{1} \geq 1 / 2, \alpha_{2} \leq 2 / 3, \beta_{2} \geq$ $\pi / 4=0.7853 \ldots, \alpha_{3} \leq 1 / 3, \beta_{3} \geq \sqrt{3} \log (2+\sqrt{3}) / 6=0.3801 \ldots, \alpha_{4} \leq 2 / 3$, and $\beta_{4} \geq$ $(4 \sqrt{3} \pi-9) / 18=0.7901 \ldots$

Theorem 1.3 The double inequalities

$$
\begin{gathered}
\frac{\alpha_{5}}{H(a, b)}+\frac{1-\alpha_{5}}{A(a, b)}<\frac{1}{N_{A H}(a, b)}<\frac{\beta_{5}}{H(a, b)}+\frac{1-\beta_{5}}{A(a, b)}, \\
\frac{\alpha_{6}}{H(a, b)}+\frac{1-\alpha_{6}}{A(a, b)}<\frac{1}{N_{H A}(a, b)}<\frac{\beta_{6}}{H(a, b)}+\frac{1-\beta_{6}}{A(a, b)}, \\
\frac{\alpha_{7}}{A(a, b)}+\frac{1-\alpha_{7}}{C(a, b)}<\frac{1}{N_{C A}(a, b)}<\frac{\beta_{7}}{A(a, b)}+\frac{1-\beta_{7}}{C(a, b)}, \\
\frac{\alpha_{8}}{A(a, b)}+\frac{1-\alpha_{8}}{C(a, b)}<\frac{1}{N_{A C}(a, b)}<\frac{\beta_{8}}{A(a, b)}+\frac{1-\beta_{8}}{C(a, b)},
\end{gathered}
$$

hold for all $a, b>0$ with $a \neq b$ if and only if $\alpha_{5} \leq 0, \beta_{5} \geq 2 / 3, \alpha_{6} \leq 0, \beta_{6} \geq 1 / 3, \alpha_{7} \leq[2 \sqrt{3}-$ $\log (2+\sqrt{3})] /[2 \sqrt{3}+\log (2+\sqrt{3})]=0.4490 \ldots, \beta_{7} \geq 2 / 3, \alpha_{8} \leq(9 \sqrt{3}-4 \pi) /(3 \sqrt{3}+4 \pi)=$ $0.1701 \ldots$, and $\beta_{8} \geq 1 / 3$.

\section{Lemmas}

In order to prove our main results we need several lemmas, which we present in this section.

Lemma 2.1 (See [15, Theorem 1.25]) For $-\infty<a<b<\infty$, let $f, g:[a, b] \rightarrow \mathbb{R}$ be continuous on $[a, b]$, and be differentiable on $(a, b)$, let $g^{\prime}(x) \neq 0$ on $(a, b)$. If $f^{\prime}(x) / g^{\prime}(x)$ is increasing (decreasing) on $(a, b)$, then so are

$$
\frac{f(x)-f(a)}{g(x)-g(a)} \quad \text { and } \quad \frac{f(x)-f(b)}{g(x)-g(b)} .
$$

If $f^{\prime}(x) / g^{\prime}(x)$ is strictly monotone, then the monotonicity in the conclusion is also strict.

Lemma 2.2 (See [16, Lemma 1.1]) Suppose that the power series $f(x)=\sum_{n=0}^{\infty} a_{n} x^{n}$ and $g(x)=\sum_{n=0}^{\infty} b_{n} x^{n}$ have the radius of convergence $r>0$ and $a_{n}, b_{n}>0$ for all $n \geq 0$. If the sequence $\left\{a_{n} / b_{n}\right\}$ is (strictly) increasing (decreasing) for all $n \geq 0$, then the function $f(x) / g(x)$ is also (strictly) increasing (decreasing) on $(0, r)$.

Lemma 2.3 (See [12, Theorem 4.1]) If $a>b$, then

$$
N(b, a)>N(a, b) .
$$

Lemma 2.4 The function

$$
\varphi_{1}(t)=\frac{\sinh (2 t)-4 \sinh (t)+2 t}{\sinh (2 t)-2 \sinh (t)}
$$

is strictly increasing from $(0, \infty)$ onto $(2 / 3,1)$. 
Proof Making use of power series expansion we get

$$
\varphi_{1}(t)=\frac{\sum_{n=1}^{\infty} \frac{2^{2 n+1}-4}{(2 n+1) !} t^{2 n+1}}{\sum_{n=1}^{\infty} \frac{2^{2 n+1}-2}{(2 n+1) !} t^{2 n+1}}=\frac{\sum_{n=0}^{\infty} \frac{2^{2 n+3}-4}{(2 n+3) !} t^{2 n}}{\sum_{n=0}^{\infty} \frac{2^{2 n+3}-2}{(2 n+3) !} t^{2 n}} .
$$

Let

$$
a_{n}=\frac{2^{2 n+3}-4}{(2 n+3) !}, \quad b_{n}=\frac{2^{2 n+3}-2}{(2 n+3) !} .
$$

Then

$$
a_{n}>0, \quad b_{n}>0,
$$

and $a_{n} / b_{n}=1-1 /\left(2^{2 n+2}-1\right)$ is strictly increasing for all $n \geq 0$.

Note that

$$
\varphi_{1}\left(0^{+}\right)=\frac{a_{0}}{b_{0}}=\frac{2}{3}, \quad \varphi_{1}(\infty)=\lim _{n \rightarrow \infty} \frac{a_{n}}{b_{n}}=1 .
$$

Therefore, Lemma 2.4 follows easily from Lemma 2.2 and (2.1)-(2.4) together with the monotonicity of the sequence $\left\{a_{n} / b_{n}\right\}$.

Lemma 2.5 The function

$$
\varphi_{2}(t)=\frac{2 t-\sin (2 t)}{\sin t(1-\cos t)}
$$

is strictly increasing from $(0, \pi / 2)$ onto $(8 / 3, \pi)$.

Proof Let $f_{1}(t)=2 t-\sin (2 t)$ and $g_{1}(t)=\sin t(1-\cos t)$. Then simple computations lead to

$$
\varphi_{2}(t)=\frac{f_{1}(t)-f_{1}(0)}{g_{1}(t)-g_{1}(0)}
$$

and $f_{1}^{\prime}(t) / g_{1}^{\prime}(t)=4[1-1 /(2+1 / \cos t)]$ is strictly increasing on $(0, \pi / 2)$.

Note that

$$
\varphi_{2}\left(0^{+}\right)=\lim _{t \rightarrow 0^{+}} \frac{f_{1}^{\prime}(t)}{g_{1}^{\prime}(t)}=\frac{8}{3}, \quad \varphi_{2}(\pi / 2)=\pi .
$$

Therefore, Lemma 2.5 follows from Lemma 2.1, (2.5), (2.6), and the monotonicity of $f_{1}^{\prime}(t) / g_{1}^{\prime}(t)$.

Lemma 2.6 The function

$$
\varphi_{3}(t)=\frac{\sinh (t) \cosh (t)-t}{[\sinh (t) \cosh (t)+t](\cosh (t)-1)}
$$

is strictly decreasing from $(0, \infty)$ onto $(0,2 / 3)$. 
Proof Simple computations lead to

$$
\begin{aligned}
\varphi_{3}(t) & =\frac{2 \sinh (2 t)-4 t}{\sinh (3 t)+4 t \cosh (t)+\sinh (t)-2 \sinh (2 t)-4 t} \\
& =\frac{\sum_{n=0}^{\infty} \frac{2^{2 n+4}}{(2 n+3) !} t^{2 n}}{\sum_{n=0}^{\infty} \frac{3^{2 n+3}-2^{2 n+4}+8 n+13}{(2 n+3) !} t^{2 n}} .
\end{aligned}
$$

Let

$$
a_{n}=\frac{2^{2 n+4}}{(2 n+3) !}, \quad b_{n}=\frac{3^{2 n+3}-2^{2 n+4}+8 n+13}{(2 n+3) !} .
$$

Then

$$
a_{n}>0, \quad b_{n}>0,
$$

and

$$
\frac{a_{n+1}}{b_{n+1}}-\frac{a_{n}}{b_{n}}=-\frac{2^{2 n+4}\left(5 \times 3^{2 n+3}-24 n-31\right)}{\left(3^{2 n+5}-2^{2 n+6}+8 n+21\right)\left(3^{2 n+3}-2^{2 n+4}+8 n+13\right)}<0
$$

for all $n \geq 0$.

Note that

$$
\varphi_{3}\left(0^{+}\right)=\frac{a_{0}}{b_{0}}=\frac{2}{3}, \quad \varphi_{3}(\infty)=\lim _{n \rightarrow \infty} \frac{a_{n}}{b_{n}}=0 .
$$

Therefore, Lemma 2.6 follows easily from (2.7)-(2.11) and Lemma 2.2.

Lemma 2.7 The function

$$
f(t)=9 \cos t+\frac{t}{\sin t}
$$

is strictly decreasing on the interval $(0, \pi / 2)$.

Proof Let $f_{2}(t)=9 \sin t \cos t+t$ and $g_{2}(t)=\sin t$. Then simple computations lead to

$$
\begin{aligned}
& f(t)=\frac{f_{2}(t)-f_{2}(0)}{g_{2}(t)-g_{2}(0)}, \\
& \frac{f_{2}^{\prime}(t)}{g_{2}^{\prime}(t)}=\frac{18 \cos ^{2} t-8}{\cos t},
\end{aligned}
$$

and

$$
\left[\frac{f_{2}^{\prime}(t)}{g_{2}^{\prime}(t)}\right]^{\prime}=-\frac{2 \sin t\left(9 \cos ^{2} t+4\right)}{\cos ^{2} t}<0
$$

for $t \in(0, \pi / 2)$.

Therefore, Lemma 2.7 follows easily from (2.12) and (2.13) together with Lemma 2.1. 
Lemma 2.8 The function

$$
\varphi_{4}(t)=\frac{\sin t \cos t-t}{(t+\sin t \cos t)(1-\cos t)}
$$

is strictly decreasing from $(0, \pi / 2)$ onto $(-1,-2 / 3)$.

Proof Let $f_{3}(t)=\sin t \cos t-t$ and $g_{3}(t)=(t+\sin t \cos t)(1-\cos t)$. Then simple computations lead to

$$
\begin{aligned}
& \varphi_{4}(t)=\frac{f_{3}(t)}{g_{3}(t)}=\frac{f_{3}(t)-f_{3}(0)}{g_{3}(t)-g_{3}(0)}, \\
& \frac{f_{3}^{\prime}(t)}{g_{3}^{\prime}(t)}=\frac{f_{3}^{\prime}(t)-f_{3}^{\prime}(0)}{g_{3}^{\prime}(t)-g_{3}^{\prime}(0)},
\end{aligned}
$$

and

$$
\frac{f_{3}^{\prime \prime}(t)}{g_{3}^{\prime \prime}(t)}=\frac{4}{4-\left(9 \cos t+\frac{t}{\sin t}\right)} .
$$

Note that

$$
\varphi_{4}\left(0^{+}\right)=\lim _{t \rightarrow 0^{+}} \frac{f_{3}^{\prime \prime}(t)}{g_{3}^{\prime \prime}(t)}=-\frac{2}{3}, \quad \varphi_{4}\left(\frac{\pi}{2}\right)=-1 .
$$

Therefore, Lemma 2.8 follows from Lemma 2.1 and Lemma 2.7 together with (2.14)(2.17).

\section{Proofs of Theorems 1.1-1.3}

Proof of Theorem 1.1 It follows from (1.1)-(1.3) as we clearly see that

$$
\begin{aligned}
N_{A H}(a, b) & =\frac{1}{2}\left[A(a, b)+\frac{H^{2}(a, b)}{S_{A H}(a, b)}\right]=\frac{1}{2} A(a, b)\left[1+\left(1-v^{2}\right)^{2} \frac{p}{\tanh (p)}\right] \\
& =\frac{1}{2} A(a, b)\left[1+\frac{p}{\tanh (p) \cosh ^{2}(p)}\right]=\frac{1}{2} A(a, b)\left[1+\frac{2 p}{\sinh (2 p)}\right], \\
N_{H A}(a, b) & =\frac{1}{2}\left[H(a, b)+\frac{A^{2}(a, b)}{S_{H A}(a, b)}\right]=\frac{1}{2} A(a, b)\left[\left(1-v^{2}\right)+\frac{q}{\sin q}\right] \\
& =\frac{1}{2} A(a, b)\left[\cos q+\frac{q}{\sin q}\right], \\
N_{C A}(a, b) & =\frac{1}{2}\left[C(a, b)+\frac{A^{2}(a, b)}{S_{C A}(a, b)}\right]=\frac{1}{2} A(a, b)\left[\left(1+v^{2}\right)+\frac{r}{\sinh (r)}\right] \\
& =\frac{1}{2} A(a, b)\left[\cosh (r)+\frac{r}{\sinh (r)}\right], \\
N_{A C}(a, b) & =\frac{1}{2}\left[A(a, b)+\frac{C^{2}(a, b)}{S_{A C}(a, b)}\right]=\frac{1}{2} A(a, b)\left[1+\left(1+v^{2}\right)^{2} \frac{s}{\tan (s)}\right] \\
& =\frac{1}{2} A(a, b)\left[1+\frac{s}{\tan (s) \cos ^{2} s}\right]=\frac{1}{2} A(a, b)\left[1+\frac{2 s}{\sin (2 s)}\right] .
\end{aligned}
$$


Inequalities (1.8) follow easily from $H(a, b)<A(a, b)<C(a, b)$ and Lemma 2.3 together with the fact that $N_{K L}(a, b)$ is a mean of $K(a, b)$ and $L(a, b)$ for $K(a, b), L(a, b) \in$ $\{H(a, b), A(a, b), C(a, b)\}$.

Proof of Theorem 1.2 Without loss of generality, we assume that $a>b$. Let $v=(a-b) /$ $(a+b) \in(0,1), p \in(0, \infty), q \in(0, \pi / 2), r \in(0, \log (2+\sqrt{3}))$, and $s \in(0, \pi / 3)$ be the parameters such that $1 / \cosh (p)=\cos (q)=1-v^{2}, \cosh (r)=\sec (s)=1+v^{2}$. Then from (1.4)-(1.7) we have

$$
\begin{aligned}
\frac{N_{A H}(a, b)-H(a, b)}{A(a, b)-H(a, b)} & =\frac{[1+2 p / \sinh (2 p)] / 2-\left(1-v^{2}\right)}{v^{2}} \\
& =\frac{[1+2 p / \sinh (2 p)] / 2-1 / \cosh (p)}{1-1 / \cosh (p)}=\varphi_{1}(p), \\
\frac{N_{H A}(a, b)-H(a, b)}{A(a, b)-H(a, b)} & =\frac{[\cos q+q / \sin q] / 2-\left(1-v^{2}\right)}{v^{2}} \\
& =\frac{[\cos q+q / \sin q] / 2-\cos q}{1-\cos q}=\frac{1}{4} \varphi_{2}(q), \\
\frac{N_{C A}(a, b)-A(a, b)}{C(a, b)-A(a, b)} & =\frac{[\cosh (r)+r / \sinh (r)] / 2-1}{v^{2}} \\
& =\frac{[\cosh (r)+r / \sinh (r)] / 2-1}{\cosh (r)-1}=\frac{1}{2} \varphi_{1}(r), \\
\frac{N_{A C}(a, b)-A(a, b)}{C(a, b)-A(a, b)} & =\frac{[1+2 s / \sin (2 s)] / 2-1}{v^{2}} \\
& =\frac{[1+2 s / \sin (2 s)] / 2-1}{\sec (s)-1}=\frac{1}{4} \varphi_{2}(s),
\end{aligned}
$$

where the functions $\varphi_{1}$ and $\varphi_{2}$ are defined as in Lemmas 2.4 and 2.5, respectively.

Note that

$$
\varphi_{1}[\log (2+\sqrt{3})]=\sqrt{3} \log (2+\sqrt{3}) / 6
$$

and

$$
\varphi_{2}\left(\frac{\pi}{3}\right)=\frac{8 \sqrt{3} \pi-18}{9} .
$$

Therefore, inequality (1.9) holds for all $a, b>0$ with $a \neq b$ if and only if $\alpha_{1} \leq 1 / 3$ and $\beta_{1} \geq 1 / 2$ follows from (3.1) and Lemma 2.4, inequality (1.10) holds for all $a, b>0$ with $a \neq b$ if and only if $\alpha_{2} \leq 2 / 3$ and $\beta_{2} \geq \pi / 4$ follows from (3.2) and Lemma 2.5, inequality (1.11) holds for all $a, b>0$ with $a \neq b$ if and only if $\alpha_{3} \leq 1 / 3$ and $\beta_{3} \geq \sqrt{3} \log (2+\sqrt{3}) / 6$ follows from (3.3) and (3.5) together with Lemma 2.4, and inequality (1.12) holds for all $a, b>0$ with $a \neq b$ if and only if $\alpha_{4} \leq 2 / 3$ and $\beta_{4} \geq(4 \sqrt{3} \pi-9) / 18$ follows from (3.4) and (3.6) together with Lemma 2.5 .

Proof of Theorem 1.3 Without loss of generality, we assume that $a>b$. Let $v=(a-b)$ / $(a+b) \in(0,1), p \in(0, \infty), q \in(0, \pi / 2), r \in(0, \log (2+\sqrt{3}))$, and $s \in(0, \pi / 3)$ be the parameters such that $1 / \cosh (p)=\cos (q)=1-v^{2}, \cosh (r)=\sec (s)=1+v^{2}$. Then from (1.4)-(1.7) 
we have

$$
\begin{aligned}
& \frac{1 / N_{A H}(a, b)-1 / A(a, b)}{1 / H(a, b)-1 / A(a, b)}=\frac{\frac{2}{1+2 p / \sinh (2 p)}-1}{\frac{1}{1-v^{2}}-1}=\frac{\frac{2 \sinh (2 p)}{2 p+\sinh (2 p)}-1}{\cosh (p)-1}=\varphi_{3}(p), \\
& \frac{1 / N_{H A}(a, b)-1 / A(a, b)}{1 / H(a, b)-1 / A(a, b)}=\frac{\frac{2}{\cos (q)+q / \sin (q)}-1}{\frac{1}{1-v^{2}}-1}=\frac{\frac{2 \sin (q)-\sin (q) \cos (q)-q}{\sin (q) \cos (q)+q}}{\frac{1-\cos (q)}{\cos (q)}}=1+\varphi_{4}(q), \\
& \frac{1 / N_{C A}(a, b)-1 / C(a, b)}{1 / A(a, b)-1 / C(a, b)}=\frac{\frac{2}{\cosh (r)+r / \sinh (r)}-\frac{1}{1+v^{2}}}{1-\frac{1}{1+v^{2}}}=\varphi_{3}(r),
\end{aligned}
$$

and

$$
\frac{1 / N_{A C}(a, b)-1 / C(a, b)}{1 / A(a, b)-1 / C(a, b)}=1+\varphi_{4}(s)
$$

where the functions $\varphi_{3}$ and $\varphi_{4}$ are defined as in Lemmas 2.6 and 2.8, respectively.

Note that

$$
\varphi_{3}[\log (2+\sqrt{3})]=\frac{2 \sqrt{3}-\log (2+\sqrt{3})}{2 \sqrt{3}+\log (2+\sqrt{3})}
$$

and

$$
\varphi_{4}\left(\frac{\pi}{3}\right)=-\frac{8 \pi-6 \sqrt{3}}{4 \pi+3 \sqrt{3}} .
$$

Therefore, Theorem 1.3 follows easily from (3.7)-(3.12) together with Lemmas 2.6 and 2.8 .

\section{Competing interests}

The authors declare that they have no competing interests.

\section{Authors' contributions}

All authors contributed equally to the writing of this paper. All authors read and approved the final manuscript.

\section{Author details}

'School of Mathematics and Computation Science, Hunan City University, Yiyang, 413000, China. ${ }^{2}$ Department of Mathematics, Huzhou Teachers College, Huzhou, 313000, China. ${ }^{3}$ School of Mathematics and Econometrics, Hunan University, Changsha, 410082, China.

\section{Acknowledgements}

This research was supported by the Natural Science Foundation of China under Grants 61374086, 11371125, and 11401192, the Natural Science Foundation of Hunan Province under Grant 12C0577, and the Research Foundation of Education Bureau of Hunan Province under Grant 14A026.

\section{Received: 21 May 2014 Accepted: 3 September 2014 Published: 25 Sep 2014}

\section{References}

1. Carlson, BC: Special Functions of Applied Mathematics. Academic Press, New York (1977)

2. Carlson, BC, Gustafson, JL: Asymptotic approximations for symmetric elliptic integrals. SIAM J. Math. Anal. 25(2), 288-303 (1994)

3. Neuman, E, Sándor, J: On the Schwab-Borchardt mean. Math. Pannon. 14(2), 253-266 (2003)

4. Neuman, E, Sándor, J: On the Schwab-Borchardt mean II. Math. Pannon. 17(1), 49-59 (2006)

5. Neuman, E: Inequalities for the Schwab-Borchardt mean and their applications. J. Math. Inequal. 5(4), 601-609 (2011)

6. Carlson, BC: Algorithms involving arithmetic and geometric means. Am. Math. Mon. 78, 496-505 (1971)

7. Brenner, JL, Carlson, BC: Homogeneous mean values: weights and asymptotics. J. Math. Anal. Appl. 123(1), 265-280 (1987) 
8. He, Z-Y, Chu, Y-M, Wang, M-K: Optimal bounds for Neuman means in terms of harmonic and contraharmonic means. J. Appl. Math. 2013, Article ID 807623 (2013)

9. Chu, Y-M, Qian, W-Q: Refinements of bounds for Neuman means. Abstr. Appl. Anal. 2014, Article ID 354132 (2014)

10. Neuman, E: On some means derived from the Schwab-Borchardt mean. J. Math. Inequal. 8(1), 171-183 (2014)

11. Neuman, E: On some means derived from the Schwab-Borchardt mean II. J. Math. Inequal. 8(2), 361-370 (2014)

12. Neuman, E: On a new bivariate mean. Aequ. Math. (2013). doi:10.1007/s00010-013-0224-8

13. Zhang, Y, Chu, Y-M, Jiang, Y-L: Sharp geometric mean bounds for Neuman means. Abstr. Appl. Anal. 2014, Article ID $949815(2014)$

14. Guo, Z-J, Zhang, Y, Chu, Y-M, Song, Y-Q: Sharp bounds for Neuman means in terms of geometric, arithmetic and quadratic means. arXiv:1405.4384v

15. Anderson, GD, Qiu, S-L, Vamanamurthy, MK, Vuorinen, M: Generalized elliptic integrals and modular equations. Pac. J. Math. 192(1), 1-37 (2000)

16. Simić, S, Vuorinen, M: Landen inequalities for zero-balanced hypergeometric functions. Abstr. Appl. Anal. 2012 Article ID 932061 (2012)

10.1186/1029-242X-2014-370

Cite this article as: Chen et al.: Note on certain inequalities for Neuman means. Journal of Inequalities and Applications $2014,2014: 370$

\section{Submit your manuscript to a SpringerOpen ${ }^{\circ}$ journal and benefit from:}

- Convenient online submission

- Rigorous peer review

- Immediate publication on acceptance

- Open access: articles freely available online

- High visibility within the field

- Retaining the copyright to your article 